\title{
用于目标跟踪的孪生渐进注意引导融合网络
}

\author{
范颖, 宋晓宁 ${ }^{*}$ \\ (江南大学人工智能与计算机学院 无锡 214122) \\ (x.song@jiangnan.edu.cn)
}

\begin{abstract}
摘 要：针对基于孪生网络的目标跟踪中大部分方法是利用主干网络的最后一层语义特征来计算相似度，而单一地 利用深层特征空间往往是不够的问题，提出基于孪生网络的渐进注意引导融合跟踪方法. 首先采用主干网络提取深 层和浅层特征信息; 然后通过特征聚合模块，以自顶向下的方法去编码融合深层语义信息以及浅层空间结构信息， 并利用注意力模块减少融合产生的特征元余; 最后计算目标和搜索区域的匹配相似度, 以进行目标跟踪. 在加人注 意力模块后, 跟踪器可以选择性地整合多层特征信息, 提升了跟踪器的性能. 在 OTB2013, OTB50, OTB2015, VOT2016 以及 VOT2017 这 5 个公共基准数据库上，与 SiamDW 等方法进行实验的结果表明，文中方法能够有效地提 升跟踪的精度及成功率.
\end{abstract}

关键词：目标跟踪；多层融合; 注意机制; 孪生网络

中图法分类号: TP391.41 DOI: 10.3724/SP.J.1089.2021.18392

\section{Siamese Progressive Attention-Guided Fusion Network for Object Tracking}

\author{
Fan Ying and Song Xiaoning* \\ (School of Artificial Intelligence and Computer Science, Jiangnan University, Wuxi 214122)
}

\begin{abstract}
For the most of object tracking algorithms using siamese networks, the semantic feature derived from the last layer of the backbone network is used to calculate the similarity. However, the use of single deep feature space often leads to partial loss of effective information. To address this issue, the siamese progressive attention-guided fusion network is proposed. First, the deep and shallow feature information is simultaneously extracted using the backbone network. Second, a top-down strategy is adopted to gradually encode and fuse deep semantic information, as well as shallow spatial structure information is obtained from the progressive feature aggregation module. We then use attention module to reduce feature redundancy that generated by fusion. Last, the optimal solution of object tracking is formed by calculating the similarity between the target and search area. By means of attention module, the tracker can selectively integrate multi-level features information to enhance the performance of the applications. As compared with SiamDW and other traditional methods, experimental results conducted on the five common tracking benchmarks including OTB2013, OTB50, OTB2015, VOT2016 and VOT2017, demonstrate that the effectiveness of the proposed algorithm in terms of tracking accuracy and success rate.
\end{abstract}

Key words: object tracking; multi-level fusion; attention mechanism; siamese network

收稿日期：2020-05-18; 修回日期：2020-09-30. 基金项目：国家重点研发计划子课题(2017YFC1601800); 国家自然科学基金 (61876072); 中国博士后科学基金特别资助项目(2018T110441); 江苏省六大人才高峰项目(XYDXX-012). 范颖(1996一), 男, 硕士研 究生, 主要研究方向为计算机图像处理、深度学习; 宋晓宁(1975-), 男, 博士, 教授, 博士生导师, CCF 会员, 论文通讯作者, 主要研 究方向为人工智能与模式识别. 
视觉目标跟踪是计算机视觉的基本问题之一, 近年来得到越来越多的关注. 它在视频监控 ${ }^{[1]}$ 、人 机交互 ${ }^{[2]}$ 以及视频编辑 ${ }^{[3]}$ 等方面有着广泛的应用. 但由于尺度变化、遮挡、变形、快速运动和背景杂 波等实际因素的影响, 视觉目标跟踪仍然具有很 大的挑战性.

大多数现有的跟踪方法都是基于相关滤波器 (correlation filter, $\mathrm{CF})^{[4-11]}$ 和孪生网络这 2 个成功的 框架开发的.

基于相关滤波的方法由于使用快速傅里叶变 换, 具有较高的计算效率和不错的跟踪精度. 例 如, $\operatorname{MOSSE}^{[4]}$ 是首个将相关滤波器用于目标跟踪 的方法, 其运行速度约为 700 帧/s. 然而对于复杂 的跟踪场景, 该跟踪器的性能通常会显著下降. 所 以为了应对各种挑战和实现更好的跟踪性能，相 关滤波跟踪器之后的发展集中在核函数 ${ }^{[5]}$ 、运动信 息 ${ }^{[6]}$ 、多维特征 ${ }^{[7]}$ 、多尺度估计 ${ }^{[8]}$ 、减缓边界效应 ${ }^{[9]}$ 和深度卷积特征 ${ }^{[10-11]}$ 等方面. 尤其是深度卷积特 征, 它与手工特征相比具有更强的表示能力, 已成 为提高跟踪精度的重要手段. 随着这些跟踪技术 的发展，在各种跟踪基准及挑战 ${ }^{[2-15]}$ 上，基于相关 滤波跟踪器的精度开始有了大幅提高. 不过也随 之带来了相当大的计算开销, 导致跟踪器不能满 足实时性的要求 (大于 30 帧/s).

为了平衡跟踪性能和速度，基于孪生网络的 目标跟踪 ${ }^{[16-27]}$ 成为近年来流行的目标跟踪框架. 这些跟踪方法(如 SiamFC ${ }^{[16]}$ )使用孪生卷积网络提 取目标区域和搜索区域的特征表示进行比较，从 而将跟踪问题转化为匹配学习问题. 由于该框架 是端到端训练的，更容易在跟踪数据集上进行训 练, 从而使得这些跟踪方法既可以提供高精度, 又 无需任何微调或在线更新过程. 最近几个扩展在 孪生网络进行特征提取后, 使用区域建议网络 (region proposal network, RPN) ${ }^{[28]}$ 进行分类和检测, 在各种基准数据集上也都取得了优异的结果.

然而，视觉目标跟踪仍然受到几个问题的困 扰. 首先, 这些跟踪方法的识别能力在很大程度上 取决于孪生网络的特征提取能力. 其次, 在孪生网 络中, 往往都是从最后一层卷积层中提取特征来进 行匹配相似度计算. 而深层特征表示往往分辨率较 低, 不利于准确定位. 为解决上述问题, 本文开发 了一种端到端深度模型架构, 名为孪生渐进注意引 导融合网络 (siamese progressive attention-guided fusion network, SiamPAGF). 它采用了先进的主干 网络, 以获得更好的特征识别能力. 另外, 主干网
络的深层特征表示尽管分辨率低，但其语义层次 高, 可以有效地区分不同类别的目标; 浅层特征表 示分辨率高, 可以捕获丰富的结构细节信息, 对于 目标精确定位是非常有用的. 所以本文采用自顶 向下的方式逐步融合高层的语义信息和浅层的空 间结构信息, 并引入注意力模块来细化特征抑制 融合噪声, 逐步提高网络的特征学习能力. 通过本 文方法, 可以学习到更强的特征表示来计算匹配 相似度, 有效地提高跟踪性能; 并在 OTB2013 ${ }^{[12]}$, OTB50, OTB2015 ${ }^{[13]}$, VOT2016 ${ }^{[14]}$ 以及 VOT2017 $7^{[15]}$ 这 5 个公共跟踪基准数据集上获得较好的跟踪结果.

\section{1 基于孪生网络的跟踪}

近些年，基于孪生网络的跟踪器引领着最新 的跟踪趋势, 因为它们能以较高的跟踪速度达到 相当高的跟踪精度. 例如, SiamFC ${ }^{[16]}$ 采用孪生网 络作为特征提取器, 将视觉目标跟踪问题表述为 一个相似度学习任务, 其首先离线训练一个相似 度量网络, 然后用它计算输人图像块和目标之间 的匹配相似度, 相似程度高的部分即跟踪目标. 该 方法结构简单且非常高效, 速度可达到 86 帧/s. 之 后, 为了使离线训练的孪生网络能够适应目标的 外观变化, DSiam ${ }^{[17]}$ 在 SiamFC 框架的基础上增加 了模型在线学习更新的能力, 即添加了 2 个线性回 归模型, 一个可以学习目标的外观变化, 另一个可 以学习抑制背景. TriSiamFC ${ }^{[18]}$ 使用三重损失代替 交叉熵损失来训练孪生网络. 为了提高孪生网络 的表示能力, $\mathrm{CFNet}^{[19]}$ 在孪生网络中引人了相关滤 波层作为可微层. RASNet ${ }^{[20]}$ 进一步引人了多种注 意机制. 另外, 为了能灵活地应对目标尺度变化, SiamRPN ${ }^{[21]}$ 引用了目标检测方向的 RPN, 将其与 孪生网络组合在一起, 使得跟踪器能够实现对目 标尺度变化的适应, 得到更精确的目标边界框, 同 时更进一步提高跟踪速度.

\section{2 本文方法}

SiamPAGF 框架如图 1 所示, 它是由孪生主干 网络、特征聚合模块和注意力模块组成. 本文方法 使用一个 3 阶段的 ResNet22 作为孪生网络的主干, 用来负责提取示例样本和搜索样本的多层特征表 示. 其中特征聚合模块应用于示例分支最后一阶 段的 3 层特征, 以自顶向下的方式进行逐步整合. 然后利用注意力模块对生成的聚合特征进行进一 
步的细化，智能地集成所需的深层浅层特征表示 信息. 最后，计算目标和搜索区域之间的匹配相似 度生成 2 个分数图, 并通过加权和组合以预测跟踪 区域.

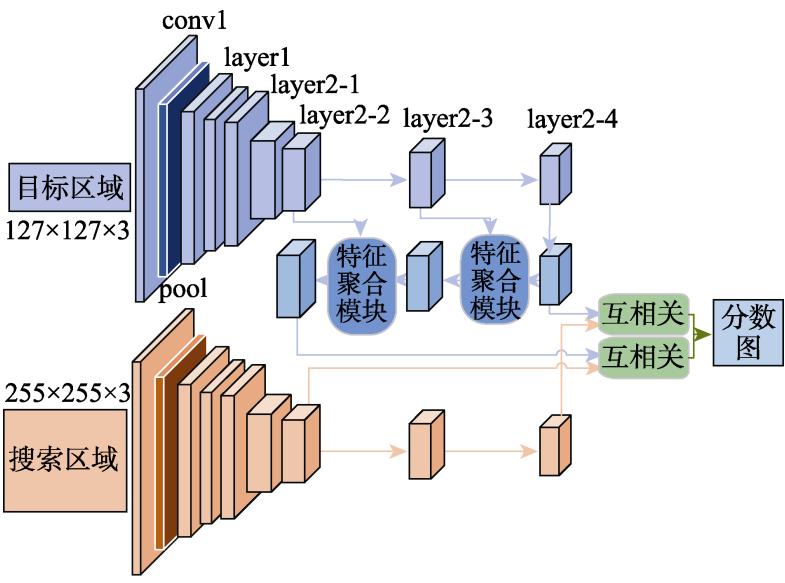

图 1 孪生渐进注意引导融合网络框架

\section{1 孪生主干网络}

众所周知，强大的特征表示对于精确和健壮 的视觉目标跟踪至关重要. 在基于孪生网络的跟 踪器中, 深度神经网络已被证明是有效的, 如 VGGNet ${ }^{[29]}$, ResNet ${ }^{[30]}$ 和 MobileNets ${ }^{[31]}$. 值得一提 的是，这些跟踪方法都是基于全卷积性质的，只适 用于所使用的主干网络没有填充操作的情况. 虽 然原始的 ResNet 可以学习到非常强大的特征表示, 但因网络中使用了填充操作，会在孪生跟踪框架 中引人位置偏差，使得目标和搜索样本的匹配相 似度下降，进而导致跟踪性能降低.

为解决这个问题, 本文提出的跟踪器采用修 改的 ResNet22 作为孪生主干网络, 这被证明是有 效的 ${ }^{[22]}$. 该网络分为 3 个阶段，总体步长为 8 , 由 22 个卷积层组成. 每当卷积层使用填充后, 都会 在之后使用裁剪操作来消除受到补零影响的特征 计算，同时保持内部块结构不变. 另外，网络在前 2 个阶段中的特征下采样是遵循原始的 ResNet. 而 在第 3 阶段, 由一个步长为 2 的最大池化来代替卷 积层执行下采样, 该层位于此阶段(总共有 4 个块, 分别为 layer2-1, layer2-2, layer2-3 和 layer2-4)的第 1 个块中.

孪生主干网络是由 2 个相同的分支组成的. 一 个称为示例分支，接收示例样本的输人; 另一个称 为搜索分支, 接收搜索样本的输人. 这 2 个分支在 卷积神经网络中共享参数, 以确保相同的转换用 于这 2 类样本.

\section{2 特征聚合模块}

由于池化、卷积等下采样操作，使得孪生主干 网络最后一层提取的特征图分辨率大大降低(如示 例分支最后一层特征图大小为 $5 \times 5$ ). 虽然深层特 征可以很好地了解语义信息, 但也导致了目标不 能够准确定位. 因此不仅需要顶层的高级特征, 还 应该将包含空间结构信息的低级特征应用到匹配 相似度计算中, 这对于最终的目标跟踪结果是十 分有利的. 然而, 本文通过研究发现独立处理每一 层特征, 即直接使用深层和浅层特征来进行计算 未必那么有效. 为此, 在网络中本文应用了特征聚 合模块，对各层信息进行逐步地编码融合，将高层 特征信息和浅层特征信息有选择性地结合在一起, 以生成综合增强的特征表示.

如图 2 所示, 特征聚合模块接收 2 个部分的输 人, 即来自对应层的低级特征和上一层通过注意 力模块 (注意力模块将在后一小节引入)产生的高 级注意特征. 本节将详细介绍高级特征和低级特 征的聚合策略，即采用级联操作将上采样后的高 级特征与低级特征融合. 具体而言, 为了级联运算 的一致性, 注意力模块(attention module, AM)生成 的高级注意特征 $\boldsymbol{S}_{l}^{\mathrm{AM}}$ 首先被送人反卷积层 $f^{\mathrm{d}}$ 中, 该反卷积层将特征上采样到与当前低级特征 $\boldsymbol{S}_{l-1}$ 相同的空间大小. 然后将该上采样的高级注意特 征和低级特征级联在一起, 并通过 $1 \times 1$ 的卷积运 算 $f^{1 \times 1}$ 进行特征通道压缩, 生成综合特征 $\tilde{\boldsymbol{S}}_{l-1}$. 此 外, 如前文所述, 深层中学习的特征包含高度抽象 的语义信息，但缺乏可以从浅层合并的细节信息. 简单地将它们融合在一起会造成特征圥余. 所以 在最后，本文将融合后的特征 $\tilde{\boldsymbol{S}}_{l-1}$ 输人到 $\mathrm{AM}$, 生 成注意特征 $\boldsymbol{S}_{l-1}^{\mathrm{AM}}$, 用以细化特征并减少噪声, 引 导特征 $\boldsymbol{S}_{l-1}^{\mathrm{AM}}$ 关注更具代表性的信息表示. 上述过 程可以描述为

$$
\begin{aligned}
\tilde{\boldsymbol{S}}_{l-1} & =f^{1 \times 1}\left(C\left(f^{\mathrm{d}}\left(\boldsymbol{S}_{l-1}^{\mathrm{AM}}\right), \boldsymbol{S}_{l-1}\right)\right), \\
\boldsymbol{S}_{l-1}^{\mathrm{AM}} & =M_{\mathrm{a}}\left(\tilde{\boldsymbol{S}}_{l-1}\right) .
\end{aligned}
$$

其中, $C(\cdot)$ 表示级联操作; $M_{\mathrm{a}}(\cdot)$ 表示 $\mathrm{AM}$.

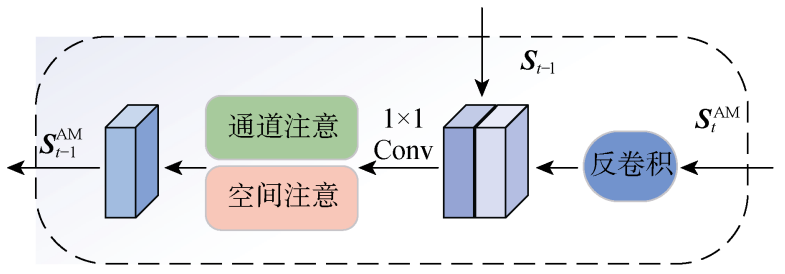

图 2 特征聚合模块细节 
接下来, 本文将生成的注意特征 $\boldsymbol{S}_{l-1}^{\mathrm{AM}}$ 作为第 $(l-2)$ 层的高级特征, 与低级特征 $\boldsymbol{S}_{l-2}$ 一起输人到 对应层次的特征聚合模块，重复上述特征融合过 程. 在网络中, 卷积特征 $\boldsymbol{S}_{l \in\{1,2,3\}}$ 分别是示例分支 网络第 3 阶段最后 3 块卷积层 layer2-2, layer2-3 和 layer2-4 的输出特征, 注意特征 $\boldsymbol{S}_{3}^{\mathrm{AM}}, \boldsymbol{S}_{2}^{\mathrm{AM}}$ 和 $\boldsymbol{S}_{1}^{\mathrm{AM}}$ 是逐层产生的. 需要注意的是, $S_{3}^{\mathrm{AM}}$ 是直接将示 例分支网络顶层特征 $\boldsymbol{S}_{3}$ 输人到 $\mathrm{AM}$ 生成的, $\boldsymbol{S}_{3}^{\mathrm{AM}}=M_{\mathrm{a}}\left(\boldsymbol{S}_{3}\right)$. 最后, 本文分别将最终生成的特 征 $\boldsymbol{S}_{1}^{\mathrm{AM}}$ 以及顶层特征 $\boldsymbol{S}_{3}$, 用来与搜索分支网络对 应层的输出特征进行匹配相似度计算, 这对最后 的跟踪效果是最优的.

\section{$2.3 \mathrm{AM}$}

如第 2.2 节所述，在特征聚合模块中，本文将 低级特征和上一层高级注意特征通过级联的方式 结合起来，得到了综合的特征表达. 该方法简单、 直观，但由于不同层次特征信息的相互矛盾，不可 避免地带来一些噪声, 影响最后的跟踪效果. 因 此, 受到文献[32-33]的启发, 本文应用一个 $\mathrm{AM}$, 用 于进一步细化特征，抑制噪声干扰，使得融合特征 更加具有代表性，如图 3 所示.

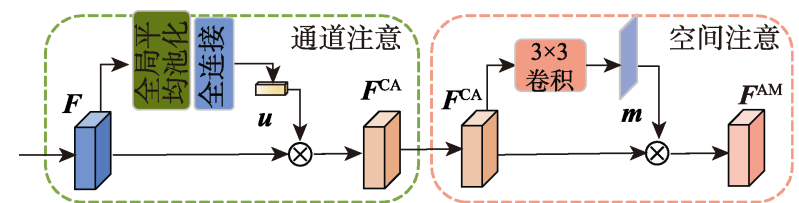

图 3 注意力模块细节

\subsection{1 空间注意机制}

近年来，注意力方法被计算机视觉方向广泛 地使用，因为其有助于关注重要特征，同时抑制不 必要的特征. 在此启发下，本文采用空间注意机制 来引导融合特征去关注更有意义的特征区域。

用符号表示方便，直接将输人特征表示为 $\boldsymbol{F} \in \mathbb{R}^{W \times H \times C}$, 其中 $C, W$ 及 $H$ 分别表示通道、宽度 和高度维度. 空间注意机制首先采用 $3 \times 3$ 卷积层 将输人特征进行压缩, 然后使用 Sigmoid 函数进行 归一化操作生成注意映射

$$
\boldsymbol{m}=\sigma\left(f^{3 \times 3}(\boldsymbol{F})\right), \boldsymbol{m} \in \mathbb{R}^{W \times H \times 1} .
$$

其中, $\sigma(\cdot)$ 是 Sigmoid 函数; $f^{3 \times 3}(\cdot)$ 表示 $3 \times 3$ 的卷 积运算. 最后, 空间注意特征(spatial attention, SA)

$$
\boldsymbol{F}^{\mathrm{SA}}=\boldsymbol{F} \otimes \boldsymbol{m}, \boldsymbol{F}^{\mathrm{SA}} \in \mathbb{R}^{W \times H \times C} .
$$

其中， $\otimes$ 表示按元素方向乘法.

\subsection{2 通道注意机制}

为缓解融合特征中的噪声干扰, 空间注意机 制是从空间的角度来处理该问题的. 事实上，由于 卷积网络特征的各个通道会对不同的语义产生响 应，本文也从通道的角度来对特征进行了强化. 例 如，通过将更大权重分配给对目标有更高反应的 特征通道, 以改进对特定目标的信息表示. 因此引 人通道注意机制是很有必要的.

通道注意机制是通过全局平均池化来压缩输 人特征的空间依赖, 然后使用 $1 \times 1$ 的卷积层和 Sigmoid 函数来突出显示对特定目标有响应的通 道. 具体来说, 给定输人特征 $\boldsymbol{F} \in \mathbb{R}^{W \times H \times C}$, 通道 注意映射为

$$
\boldsymbol{u}=\sigma\left(f^{1 \times 1}\left(P_{\mathrm{a}}(\boldsymbol{F})\right)\right), \boldsymbol{u} \in \mathbb{R}^{C \times 1 \times 1} .
$$

其中, $P_{\mathrm{a}}(\cdot)$ 是按通道划分的全局平均池化. $\boldsymbol{u}$ 作为 通道注意映射, 被应用于输人特征, 以引导生成更 具代表性的通道注意特征(channel attention, CA)

$$
\boldsymbol{F}^{\mathrm{CA}}=\boldsymbol{F} \otimes \boldsymbol{u} .
$$

本文的 AM 就是由上述的通道空间注意机制 串联组成的. 整个模块过程可表示为

$$
M_{\mathrm{a}}(\boldsymbol{F})=\boldsymbol{F}^{\mathrm{AM}}=(\boldsymbol{F} \otimes \boldsymbol{u}) \otimes \boldsymbol{m}=\boldsymbol{F}^{\mathrm{CA}} \otimes \boldsymbol{m} .
$$

\section{3 实验结果与分析}

本文的跟踪器是使用 Python 3.6 和 PyTorch 0.4.1 框架实现的. 实验是在 1 台 GeForce RTX 2080 GPU 上进行.

\section{1 实验细节}

\subsection{1 训练过程}

本文网络的训练数据来自大型目标跟踪数据 集 GOT-10k ${ }^{[34]}$, 其包含 10000 多个真实世界中移 动目标的视频片段, 分成 560 多个类别, 目标的边 界框全是手动标记完成, 总计超过 1500000 个. 本 文按照基线跟踪器 $\mathrm{SiamDW}^{[22]}$ 的方法对该数据集 进行预处理, 示例图像大小和搜索图像大小分别 为 $127 \times 127,255 \times 255$. 在训练过程中, 孪生主干网 络通过在 ImageNet 分类数据集 ${ }^{[35]}$ 上预训练的 ResNet22 模型来进行初始化. 同时使用随机梯度 下降法, 动量为 0.9 , 并将权值衰减设为 0.0005 . 学习率从 0.01 降到 0.00001 , 以指数衰减. 整个过 程包含 100 代, 同时在 4 个 GPU 上训练, 每个 GPU 最小批处理大小为 8 .

3.1.2 测试过程

在跟踪过程中, 首先根据给定的注释标签, 在 
初始帧中裁剪目标区域作为样本，然后将其调整 为 $127 \times 127$ 像素大小以输人给示例分支网络. 对 于后续的帧，以前一帧位置为中心的搜索区域被 裁剪并调整为 $255 \times 255$ 像素. 使用搜索分支网络 进行特征提取后, 将样本特征与搜索样本特征进 行相关操作, 生成大小为 $17 \times 17$ 的相似分数图. 另 外, 为了应对目标尺度变化, 本文所提出的跟踪方 法在 $1.0613^{\{-1,0,1\}}$ 这 3 个尺度上搜索目标, 并通过 以 0.3291 为因子的线性插值来更新尺度.

\section{2 与先进跟踪方法对比}

本文跟踪器 SiamPAGF 分别在 5 个公共的跟踪 基准(OTB2013，OTB50，OTB2015，VOT2016 以及 VOT2017)上, 与其他先进跟踪方法进行对比. 为 显示比较的公平, 使用这些方法作者提供的跟踪基 准结果或使用其所给的参数设置来实现这些方法.

\subsection{1 在 OTB 上评估实验}

跟踪器在 OTB 跟踪基准(OTB2013, OTB50 和 OTB2015)上进行评估，它是目前使用最广泛的公 共基准之一. 其中, OTB2013 和 OTB50 都是由 50 个完全注释的序列组成，而 OTB2015 数据集是对 OTB2013 的扩展，包含 100 个视频序列. OTB 基 准是用精度和成功率的曲线下方面积(area under curve, AUC)作为评估标准, 并采用一次性评估

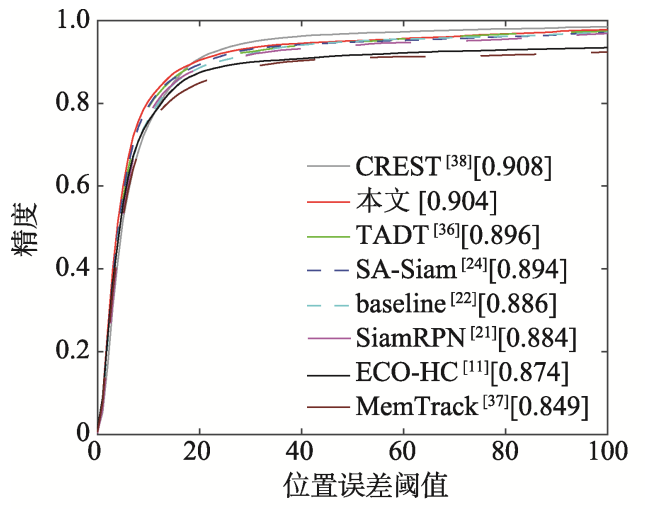

(one-pass evaluation, OPE)策略. 本文将 SiamPAGF 与许多先进跟踪器在 OTB 数据集上进行评估, 如 SiamRPN $^{[21]}$, StructSiam $^{[23]}$, SA-Siam ${ }^{[24]}$, DaSiamRPN $^{[25]}$, SiamBM ${ }^{[26]}$ 和 C-RPN ${ }^{[27]}$ 等. 具体如表 1 所示. 此外, 图 4 还显示与基线跟踪器 SiamDW 以及一些先进 跟踪方法 ${ }^{[36-38]}$ 相比，在不同的阈值下 OTB2013 和 OTB2015 基准测试的精度曲线图和成功率曲线图. 可以清楚地看出, SiamPAGF 在这 2 个数据集上每 个方面都是优于基线的.

表 1 OTB 基准下的 AUC 分数结果

\begin{tabular}{|c|c|c|c|c|}
\hline \multirow{2}{*}{ 跟踪器 } & \multicolumn{3}{|c|}{$\mathrm{AUC}$ 分数 } & \multirow{2}{*}{$\begin{array}{c}\text { 速度/ } \\
\left(\text { 帧 } \cdot \mathrm{s}^{-1}\right)\end{array}$} \\
\hline & OTB2013 & OTB50 & OTB2015 & \\
\hline SiamFC $^{[16]}$ & 0.607 & 0.516 & 0.582 & 86.0 \\
\hline $\operatorname{DSiam}^{[17]}$ & 0.656 & & & 25.0 \\
\hline $\operatorname{RASNet}^{[20]}$ & 0.670 & & 0.642 & 83.0 \\
\hline $\operatorname{SiamRPN}^{[21]}$ & 0.658 & 0.592 & 0.637 & 200.0 \\
\hline StructSiam $^{[23]}$ & 0.637 & & 0.621 & 45.0 \\
\hline SA-Siam ${ }^{[24]}$ & 0.676 & 0.610 & 0.657 & 50.0 \\
\hline $\operatorname{DaSiamRPN}^{[25]}$ & 0.656 & 0.602 & 0.658 & 160.0 \\
\hline $\operatorname{SiamBM}^{[26]}$ & 0.684 & 0.617 & 0.662 & 48.0 \\
\hline $\mathrm{C}-\mathrm{RPN}^{[27]}$ & 0.675 & & 0.663 & 32.0 \\
\hline SiamDW-FC ${ }^{[22]}$ & 0.663 & & 0.654 & 70.0 \\
\hline 本文 & 0.691 & 0.638 & 0.671 & 89.0 \\
\hline
\end{tabular}

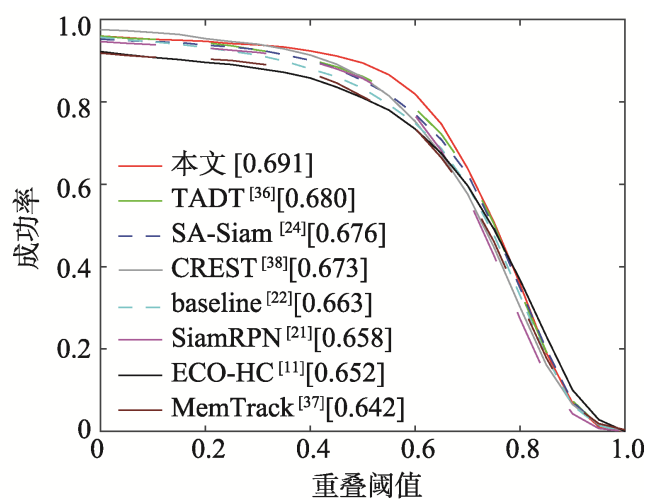

aTB2013数据集

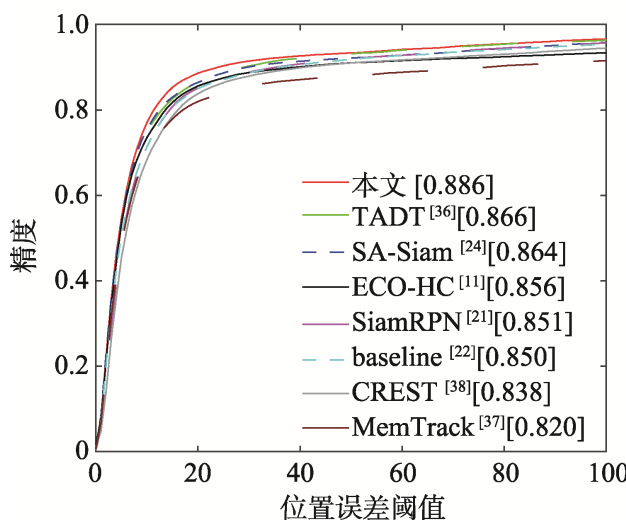

b. OTB2015数据集

图 42 个数据集上不同方法精度和成功率曲线图 


\section{2 .2 在 VOT 上评估实验}

本文还将 SiamPAGF 在 2 个 VOT 基准数据集 上进行评估，即 VOT2016 和 VOT2017。该评估通 过 VOT 官方工具包 ${ }^{[14-15]}$ 执行, 以准确度 $A$ 、鲁棒 性 $R$ 和期望平均重叠 (expected average overlap, EAO)为指标. 跟踪器在 VOT2016 和 VOT2017 数 据集上分别达到 0.59 和 0.55 的准确度, 0.22 和 0.32 的鲁棒性以及 0.378 和 0.310 的 $\mathrm{EAO}$ 分数. 在此过 程中, SiamPAGF 不仅与基于孪生网络的跟踪器比 较，还与 VOT2016 或 VOT2017 中一些排名前几位 的跟踪器进行评估. 表 2 具体显示了 VOT2016 和 VOT2017 基准上的实验结果. 可以看出, SiamPAGF 在所有这些跟踪器中都取得了非常具有竞争力的 表现.

\section{3 实验分析与讨论}

为了验证本文方法并分析每个模块的有效性,

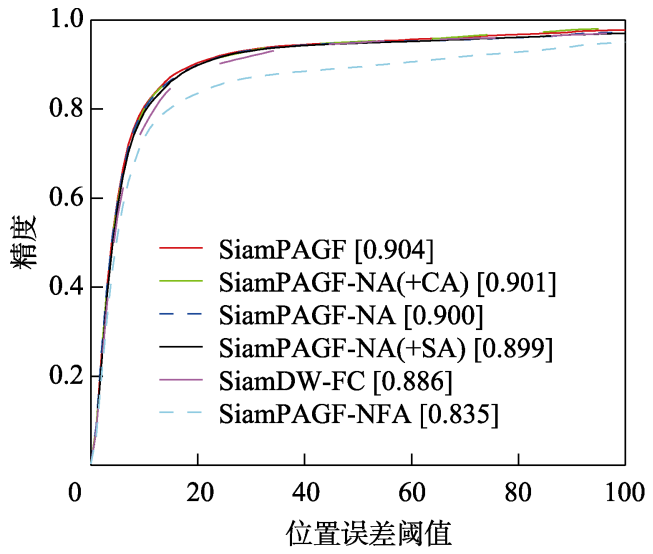

a. OTB2013数据集

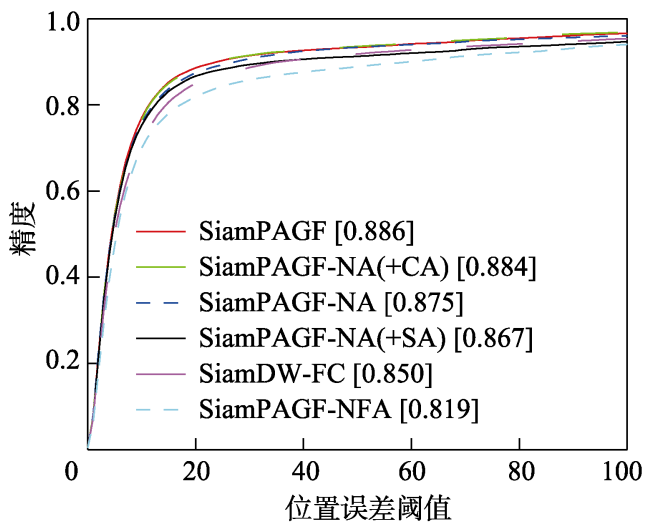

b. OTB2015数据集

图 5 基线 SiamDW 及不同变种 SiamPAGF 跟踪器精度和成功率曲线图

\subsection{1 特征聚合模块的讨论}

为研究所提出的特征聚合模块的有效性, 本文 首先实现一个没有 $\mathrm{AM}$ 的变种跟踪方法(SiamPAGF without attention module, SiamPAGF-NA), 只使用

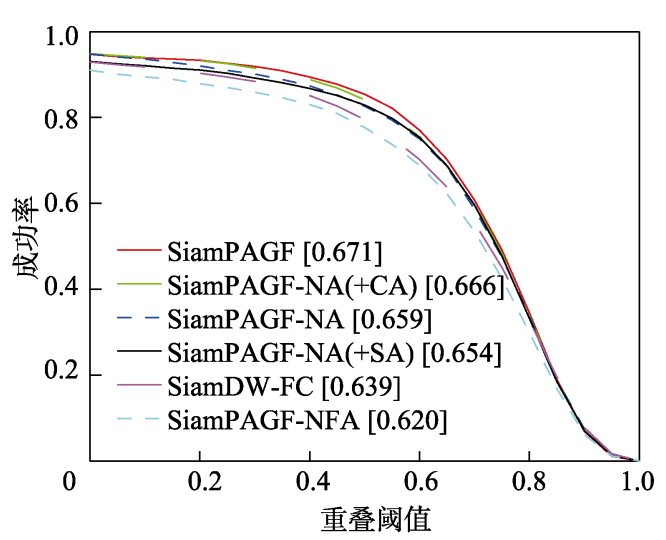

本文在 OTB 基准上评估了基线 SiamDW 以及几个 不同变种的 SiamPAGF 跟踪器. 具体实验结果可从 图 5 中直观看出.

表 2 VOT 基准评估结果

\begin{tabular}{lccccccc}
\hline \multirow{2}{*}{ 跟踪器 } & \multicolumn{3}{c}{ VOT2016 } & & \multicolumn{3}{c}{ VOT2017 } \\
\cline { 2 - 4 } \cline { 7 - 8 } & $A$ & $R$ & EAO & & $A$ & $R$ & EAO \\
\hline C-COT $^{[10]}$ & 0.54 & 0.24 & 0.331 & & & \\
MDNet $^{[40]}$ & 0.54 & 0.34 & 0.257 & & & \\
ECO $^{[11]}$ & 0.55 & 0.20 & 0.375 & 0.48 & 0.27 & 0.280 \\
LSART $^{[39]}$ & & & & 0.49 & 0.22 & 0.323 \\
SiamFC $^{[16]}$ & 0.46 & 0.53 & 0.235 & 0.50 & 0.59 & 0.188 \\
SiamRPN $^{[21]}$ & 0.56 & 0.26 & 0.344 & 0.49 & 0.46 & 0.244 \\
SA-Siam $^{[24]}$ & 0.54 & & 0.291 & 0.50 & 0.46 & 0.236 \\
DaSiamRPN & 0.61 & 0.22 & 0.411 & & 0.56 & 0.34 & 0.326 \\
SiamDW-FC & & & & 0.335 & & & 0.266 \\
本文 & 0.59 & 0.22 & 0.378 & 0.55 & 0.32 & 0.310 \\
\hline
\end{tabular}

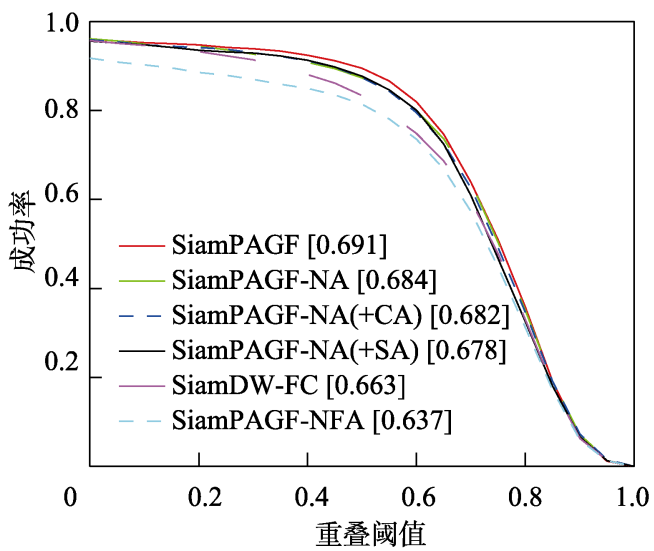

了特征聚合模块. 与基线跟踪器相比，在 OTB2013 和 OTB2015 这 2 个基准数据集上, AUC 分数提高了 0.020 和 0.007 , 分别达到 0.684 和 0.659 . 此外, 本 文还构建另一种变种跟踪器, 直接使用浅层和深层 
特征来进行相似计算(SiamPAGF without feature aggregation module, SiamPAGF-NFA). 从实验结果 可以看出，这种直接的计算方式会导致在 2 个 OTB 基准数据集的 AUC 评分显著下降, 分别为 0.639 和 0.617. 这说明本文特征聚合模块能够有效地利用 多层特征信息，对跟踪结果还是十分有用的.

\subsubsection{AM 的研究}

如第 2.3 节所述，本文方法中 AM 是从空间和 通道 2 个方面来对融合特征进行细化及抑制噪声. 为了验证该模块中不同成分对跟踪效果的个体贡 献，本文在不同的条件下进行了实验对比分析. 表 3 所示为使用不同注意机制的实验结果. 可以观察 到与跟踪器 SiamPAGF-NA 相比较, 单独集成通道 或空间注意机制的效果不如将 2 个注意机制串行 (即提出的 AM)。这说明，虽然单独的空间或通道 注意机制对最后的跟踪结果只有轻微提高甚至性 能下降, 但它们的组合能很好地改善跟踪效果.

表 3 在 OTB 基准下不同变形跟踪器的 AUC 比较

\begin{tabular}{lccc}
\hline \multicolumn{1}{c}{ 跟踪器 } & OTB2013 & OTB50 & OTB2015 \\
\hline SiamDW-FC (baseline) & 0.663 & & 0.652 \\
SiamPAGF-NFA & 0.637 & 0.562 & 0.620 \\
SiamPAGF-NA & 0.684 & 0.627 & 0.659 \\
SiamPAGF-NA(+CA) & 0.682 & 0.628 & 0.666 \\
SiamPAGF-NA(+SA) & 0.678 & 0.609 & 0.654 \\
SiamPAGF(本文) & 0.691 & 0.637 & 0.671 \\
\hline
\end{tabular}

\section{4 结 语}

本文提出一种基于孪生网络的渐进注意引导 融合跟踪方法. 通过部署强大的主干网络来捕获 表示能力丰富的特征，并使用自上而下的方式将 不同层次的卷积特征逐步调制融合起来. 另外，引 人空间通道注意机制来细化整个网络，使得网络 可以选择性地整合来自多层次的有效特征信息. 广泛地评估证明了本文方法的有效性.

\section{参考文献(References):}

[1] Liu Y C, Wang P, Wang H T. Target tracking algorithm based on deep learning and multi-video monitoring[C] //Proceedings of the 5th International Conference on Systems and Informatics. Los Alamitos: IEEE Computer Society Press, 2018: $440-444$

[2] Liu L W, Xing J L, Ai H Z, et al. Hand posture recognition using finger geometric feature[C]//Proceedings of the IEEE International Conference on Pattern Recognition. Los Ala-
Alamitos: IEEE Computer Society Press, 2012: 565-568

[3] Agarwala A, Hertzmann A, Salesin D H, et al. Keyframe-based tracking for rotoscoping and animation[J]. ACM Transactions on Graphics, 2004, 23(3): 584-591

[4] Bolme D S, Beveridge J R, Draper B A, et al. Visual object tracking using adaptive correlation filters[C] //Proceedings of the IEEE Conference on Computer Vision and Pattern Recognition. Los Alamitos: IEEE Computer Society Press, 2010: 2544-2550

[5] Henriques J F, Caseiro R, Martins P, et al. Exploiting the circulant structure of tracking-by-detection with kernels[C] //Proceedings of the 12th European Conference on Computer Vision. New York: ACM Press, 2012: 702-715

[6] Gao P, Ma Y P, Song K, et al. High performance visual tracking with circular and structural operators[J]. Knowledge-Based Systems, 2018, 161: 240-253

[7] Danelljan M, Khan F S, Felsberg M, et al. Adaptive color attributes for real-time visual tracking[C] //Proceedings of the IEEE Conference on Computer Vision and Pattern Recognition. Los Alamitos: IEEE Computer Society Press, 2014: 1090-1097

[8] Danelljan M, Häger G, Khan F S, et al. Discriminative scale space tracking $[\mathrm{J}]$. IEEE Transactions on Pattern Analysis and Machine Intelligence, 2017, 39(8): 1561-1575

[9] Danelljan M, Häger G, Khan F S, et al. Learning spatially regularized correlation filters for visual tracking[C] //Proceedings of the IEEE Conference on Computer Vision and Pattern Recognition. Los Alamitos: IEEE Computer Society Press, 2015: 4310-4318

[10] Danelljan M, Robinson A, Shahbaz Khan F, et al. Beyond correlation filters: learning continuous convolution operators for visual tracking $[\mathrm{C}] / /$ Proceedings of European Conference on Computer Vision. Heidelberg: Springer, 2016: 472-488

[11] Danelljan M, Bhat G, Shahbaz Khan F, et al. ECO: efficient convolution operators for tracking[C] //Proceedings of the IEEE Conference on Computer Vision and Pattern Recognition. Los Alamitos: IEEE Computer Society Press, 2017: 6931-6939

[12] Wu Y, Lim J, Yang M H. Online object tracking: a benchmark[C] //Proceedings of the IEEE Conference on Computer Vision and Pattern Recognition. Los Alamitos: IEEE Computer Society Press, 2013: 2411-2418

[13] Wu Y, Lim J, Yang M H. Object tracking benchmark[J]. IEEE Transactions on Pattern Analysis and Machine Intelligence, 2015, 37(9): 1834-1848

[14] Kristan M, Leonardis A, Matas J, et al. The visual object tracking VOT2016 challenge results[C] //Proceedings of European Conference on Computer Vision Workshops. Heidelberg: Springer, 2016: 777-823

[15] Kristan M, Leonardis A, Matas J, et al. The visual object tracking VOT2017 challenge results[C] //Proceedings of the IEEE International Conference on Computer Vision Workshops. Los Alamitos: IEEE Computer Society Press, 2017: 1949-1972

[16] Bertinetto L, Valmadre J, Henriques J F, et al. Fully-convolutional siamese networks for object tracking[C] // Proceedings of European Conference on Computer Vision. Heidelberg: Springer, 2016: 850-865

[17] Guo Q, Feng W, Zhou C, et al. Learning dynamic siamese 
network for visual object tracking[C] //Proceedings of the IEEE International Conference on Computer Vision Workshops. Los Alamitos: IEEE Computer Society Press, 2017: 1781-1789

[18] Dong X P, Shen J B. Triplet loss in siamese network for object tracking[C] //Proceedings of European Conference on Computer Vision. Heidelberg: Springer, 2018: 472-488

[19] Valmadre J, Bertinetto L, Henriques J F, et al. End-to-end representation learning for Correlation Filter based tracking[C] // Proceedings of the IEEE Conference on Computer Vision and Pattern Recognition. Los Alamitos: IEEE Computer Society Press, 2017: 5000-5008

[20] Wang Q, Teng Z, Xing J L, et al. Learning attentions: residual attentional siamese network for high performance online visual tracking[C] //Proceedings of the IEEE Conference on Computer Vision and Pattern Recognition. Los Alamitos: IEEE Computer Society Press, 2018: 4854-4863

[21] Li B, Yan J J, Wu W, et al. High performance visual tracking with siamese region proposal network[C] //Proceedings of the IEEE Conference on Computer Vision and Pattern Recognition. Los Alamitos: IEEE Computer Society Press, 2018: 8971-8980

[22] Zhang Z P, Peng H W. Deeper and wider siamese networks for real-time visual tracking[C] //Proceedings of the IEEE Conference on Computer Vision and Pattern Recognition. Los Alamitos: IEEE Computer Society Press, 2019: 4586-4595

[23] Zhang Y H, Wang L J, Qi J Q, et al. Structured siamese network for real-time visual tracking[C] //Proceedings of European Conference on Computer Vision. Heidelberg: Springer, 2018: $355-370$

[24] He A F, Luo C, Tian X M, et al. A twofold siamese network for real-time object tracking[C]//Proceedings of the IEEE Conference on Computer Vision and Pattern Recognition. Los Alamitos: IEEE Computer Society Press, 2018: 4834-4843

[25] Zhu Z, Wang Q, Li B, et al. Distractor-aware siamese networks for visual object tracking[C] //Proceedings of European Conference on Computer Vision. Heidelberg: Springer, 2018: 103-119

[26] He A F, Luo C, Tian X M, et al. Towards a better match in siamese network based visual object tracker[C] //Proceedings of European Conference on Computer Vision. Heidelberg: Springer, 2018: 132-147

[27] Fan H, Ling H B. Siamese cascaded region proposal networks for real-time visual tracking[C] //Proceedings of the IEEE Conference on Computer Vision and Pattern Recognition. Los Alamitos: IEEE Computer Society Press, 2019: 7944-7953

[28] Ren S Q, He K M, Girshick R, et al. Faster R-CNN: towards real-time object detection with region proposal networks[J].
IEEE Transactions on Pattern Analysis and Machine Intelligence, 2017, 39(6): 1137-1149

[29] Simonyan K, Zisserman A. Very deep convolutional networks for large-scale image recognition[OL]. [2020-05-18]. https:// arxiv.org/abs/1409.1556

[30] He K M, Zhang X Y, Ren S Q, et al. Deep residual learning for image recognition[C] //Proceedings of the IEEE Conference on Computer Vision and Pattern Recognition. Los Alamitos: IEEE Computer Society Press, 2016: 770-778

[31] Howard A G, Zhu M L, Chen B, et al. MobileNets: efficient convolutional neural networks for mobile vision applications [OL]. [2020-05-18]. https://arxiv.org/abs/1704.04861

[32] Chen L, Zhang H W, Xiao J, et al. SCA-CNN: spatial and channel-wise attention in convolutional networks for image captioning[C] //Proceedings of the IEEE Conference on Computer Vision and Pattern Recognition. Los Alamitos: IEEE Computer Society Press, 2017: 6298-6306

[33] $\mathrm{Hu}$ J, Shen L, Albanie S, et al. Squeeze-and-excitation networks[C] //Proceedings of the IEEE Conference on Computer Vision and Pattern Recognition. Los Alamitos: IEEE Computer Society Press, 2018: 7132-7141

[34] Huang L H, Zhao X, Huang K Q. GOT-10k: a large high-diversity benchmark for generic object tracking in the wild[OL]. [2020-05-18]. https://arxiv.org/abs/1810.11981

[35] Russakovsky O, Deng J, Su H, et al. ImageNet large scale visual recognition challenge[J]. International Journal of Computer Vision, 2015, 115(3): 211-252

[36] Li X, Ma C, Wu B Y, et al. Target-aware deep tracking[C] //Proceedings of the IEEE Conference on Computer Vision and Pattern Recognition. Los Alamitos: IEEE Computer Society Press, 2019: 1369-1378

[37] Yang T Y, Chan A B. Learning dynamic memory networks for object tracking[C] //Proceedings of European Conference on Computer Vision. Heidelberg: Springer, 2018: 152-167

[38] Song Y B, Ma C, Gong L J, et al. CREST: convolutional residual learning for visual tracking[C] //Proceedings of the IEEE International Conference on Computer Vision. Los Alamitos: IEEE Computer Society Press, 2017: 2574-2583

[39] Sun C, Wang D, Lu H C, et al. Learning spatial-aware regressions for visual tracking[C] //Proceedings of the IEEE Conference on Computer Vision and Pattern Recognition. Los Alamitos: IEEE Computer Society Press, 2018: 8962-8970

[40] Nam H, Han B. Learning multi-domain convolutional neural networks for visual tracking[C] //Proceedings of the IEEE Conference on Computer Vision and Pattern Recognition. Los Alamitos: IEEE Computer Society Press, 2016: 4293-4302 